\title{
Energy Revolution Path Based on Main Functional Region Planning
}

\author{
Xiaoqing Yan, Xinting Huang, and Shengyu Wu
}

\begin{abstract}
Five-Years (2016 2020) is the critical period for the industrialization and urbanization in China. The main functional region planning is promulgated as guidance for land exploitation. Therefore, higher standard for the exploitation, allocation and consumption of energy and power is necessary, which is of great importance for land exploitation. Based on the energy development principle set by main functional region planning, the regional resources and environment assessment system as well as its database is established in this paper. DRA (Delphi Relative Analysis) method is proposed to quantitatively evaluate the relative influence among factors mentioned above. Finally the advices are presented for the energy and power development of China in the medium and long term.
\end{abstract}

Index Terms-Main functional region, energy revolution, resource and environmental capacity assessment system, $13^{\text {th }}$ Five-Years.

\section{INTRODUCTION}

China enjoys a vast territory, but such characteristics as low suitability for land development, regional differences in land characteristics and fragile ecology remain noticeable. At present, our socioeconomic development is transforming from an extensive and inefficient mode into an intensive and efficient one in which it is significant to adjust and optimize the structure of land development. Therefore, the State Council unveiled the Main Functional Region Planning (hereinafter referred to as the Planning) as a guidance for land exploitation [1], [2] so as to satisfy the needs of people's livelihood, economic development and environmental protection and form a highly efficient, coordinated and sustained structure for land exploitation.

Energy development is an essential component of land exploitation [3]-[6]. At present, the problems in some areas such as excessive development of energy resources, low efficiency, unreasonable energy distribution and increasing pressure on environment and ecology become increasingly noticeable [7]-[9]. It is a must to develop energy in a proper, scientific and sustainable manner. Over the years, a number of scholars and experts have studied the structure of energy development from various perspectives of energy production and consumption [10]-[12]. According to the related provisions in the Planning, the requirements for energy development in the Planning are gathered in Section II. An assessment system of regional resources and environmental

Manuscript received October 15, 2015; revised February 28, 2016

Xiaoqing Yan and Shengyu Wu are with the Energy Research Institution of State Grid, Beijing, China (e-mail: yanxiaoqing@sgeri.sgcc.com.cn, wushengyu@sgeri.sgcc.com.cn).

Xinting Huang is with the China Power Finance Co., Ltd, Beijing, China (e-mail: dearhxt@139.com). capacity is thus established in Section III based on the fore-mentioned requirements. Delphi relative analysis of quantitative evaluation adopted in this paper is introduced in Section IV. Based on the said methods, the impact of regional resources and environmental capacity on the structure of energy development is assessed primarily and finally it is the conclusion in the last chapter.

\section{THE REQUIREMENTS OF OPTIMIZING LAND EXPLOITATION STRUCTURE FOR ENERGY DEVELOPMENT}

Energy development empowers the necessary steps of land exploitation such as post-industrialization and new urbanization and it is also an essential component in optimizing the structure of land exploitation, but it is facing a series of serious problems, i.e. lack of per capita energy resources, wasted energy due to the extensive and inefficient development mode, unsustainable supply, relatively insufficient water resources in large-scale energy bases, overlapping with ecological function areas, gradually maximized occupation of environment. In response to the problems, we should plan from a broader perspective and regulate and optimize the structure of energy development and supply based on regional development intensity and potential and regional resources and environmental capacity so as to ensure a sustainable energy supply.

According to the requirements for energy development in the Planning, we should uphold the 5 principles as follows in formulating energy planning for the $13^{\text {th }}$ Five-Years and long terms:

\section{A. Energy Basis}

Uphold a scientific principle for the layout of energy bases, thus serving the main function of the region. Since the ecological system is usually vulnerable in the areas with abundant energy resources, the aim of defining these areas as restrictive development areas is to prioritize the protection of water resources and environment instead of restricting the development of energy and mineral resources.

\section{B. Water Resources}

Strengthen the planning and management of water use based on water resources and environmental capacity to develop water resources orderly and efficiently and use them in a sustainable manner.

\section{Energy Configuration}

Regulate the development of energy bases and the construction of energy channels, control the energy flow reasonably and reduce large-scale, long-distance and inefficient energy delivery. Meanwhile, we should also 
consider the needs of urbanization pattern, the restrictions of agricultural pattern and the ecological safety pattern.

\section{Ecological Environment}

Define air pollutants and the control of total carbon emissions as rigid restrictions for energy development and include environmental space into the energy development planning so as to achieve a harmonious and sustainable development between human and nature.

\section{E. Comprehensive Exploitation}

Link the structure of energy development effectively to other steps of land exploitation and include energy into the transformation of regional socioeconomic development by planning and regulating, ensuring that energy is more than a powerhouse, but an catalyst for adjustment and transformation.

\section{THE ASSESSMENT SYSTEM OF REGIONAL RESOURCES AND ENVIRONMENTAL CAPACITY}

\section{A. The Integrated Framework}

The assessment system of regional resources and environmental capacity is established aimed at describing the necessary factors for local energy development in a comprehensive and systematic manner. Hence, the integrated framework of the assessment system is subdivided into target-level, system-level, element-level and index-level.

Target-level: quantify and evaluate the relative importance of the indexs within the whole system.

System-level: decompose the system into 2 dimensions: resource and environment. Each dimension is composed of element-levels.

Element-level: refine the 2 dimensions of the system and evaluate the factors directly related to the structure of energy development respectively.

Index-level: choose the main indexes of each element and comprehensively and systematically establish the assessment system as a guidance of energy development from a different perspective. The impact of each element on energy development can be quantified and evaluated after normalization.

\section{B. Choosing Indexes}

A comprehensive assessment system is thus established based on 2 dimensions: resource and environmental capacity in the paper, as shown in Fig. 1.

Resource capacity: the assessment system of resource capacity is established mainly based on land, water and energy resources. Firstly, for land resources, we choose the development intensity of land, farmland and forests as 3 indexes. Land development intensity is used to satisfy the needs of intensive land development. At present, industrial development is polluting farmland forests seriously and preserving sufficient farmland resources is an insuperable red line, while forests are significant in dealing with climate change. Secondly, for water resources, we choose total available water resources and shortage of water resources as 2 indexes and comprehensively evaluate water resources by absolute and relative quantities from the perspectives of total available water resources for energy sector when all water resources are equally distributed and shortage of water resources representing by the gap between surface water resources and the demand of energy development. Thirdly, for energy resources, we choose energy consumption per unit of GDP, elasticity of energy consumption and reserves of energy resources as 3 indexes. The transformation of energy consumption has higher requirements for energy consumption. And in the paper we will describe how to meet the energy demand scientifically based on the 3 indexes mentioned above.

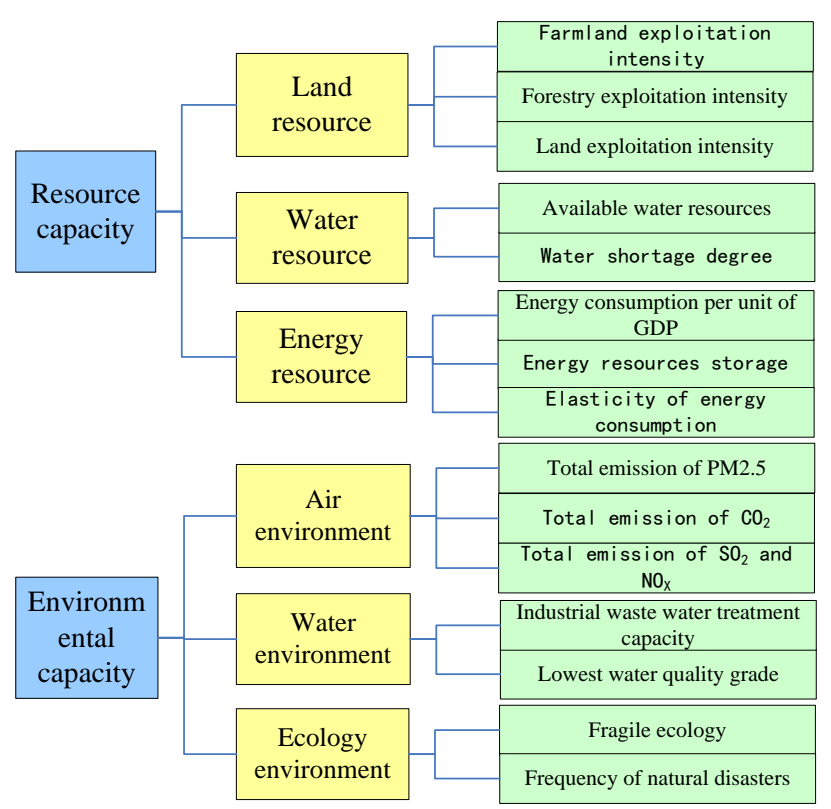

Fig. 1. The assessment system of regional resources and environmental capacity.

Environmental capacity: the assessment system of environmental capacity is established mainly based on atmospheric, water and ecological environments. Firstly, for atmospheric environment, we choose total air pollutants (fine particulate matter, $\mathrm{SO}_{2}$ and $\mathrm{NO}_{X}$ ) and total $\mathrm{CO}_{2}$ emissions as two indexes. Serious smog in the Middle East forced the governments to unveil serious policies in response. Total air pollutants have become one of the most important constraints for the development of energy sector. Moreover, only by controlling $\mathrm{CO}_{2}$ emissions can we make sure that our consumption target of non-fossil energy will be achieved. Secondly, for water environment, processing capacity of industrial waste water and quality grade of water environment are selected as two indexes. Since traditional extensive, GDP-oriented energy development pollutes water serious, the processing capacity of waste water in energy sector is an important measure to improve water quality and protect water environment. Quality grade of water environment is defined as an important criterion in managing water resources in the Action Plan of Water Pollution Prevention and Treatment formulated by the Ministry of Environmental Protection. Thirdly, ecological sensitivity and frequency of natural disasters are selected as indexes to evaluate the bearing capacity of ecological environment. Ecological sensitivity represents the disturbance of human's activity to regional environment. Important ecological sensitive areas include water resource conservation zones, natural reserve areas and 
conservation of historic sites. Frequency of natural disasters represents the possibility of force majeure. Both indexes have considerable impact on the development methods of energy bases, traversing cost of energy channels, contingency plan for emergency.

\section{QUANTITATIVE ASSESSMENT}

The reason why we choose quantitative assessment is that: on one hand, it is difficult to quantify the impact of various indexes within the assessment system on energy development, especially in different open areas. Hence, we design the quantitative assessment with the notion of "relativity" as its core; on the other hand, since the system is integrated and comprehensive, we need to reach consensus by "collective intelligence" so as to offset the evaluator's constraints in expertise and knowledge. In conclusion, quantitative assessment is established by combining Delphi method and relative analysis in this paper. Delphi relative analysis features anonymity, feedback and modesty. Its anonymity makes sure that the opinion givers can make a judgment independently, making the study more precise. Its feedback guarantees that the opinion givers can think deeply and have rights to illustrate their points in many rounds. Its modesty ensures an unanimous evaluation result.

\section{A. Two Basic Assumptions of Delphi Relative Analysis}

1) The experts can objectively evaluate their expertise and experience needed to achieve the research target and they can choose their degree of familiarity rightly in the research, giving feedback by the degrees of "familiar" "normal" and "unfamiliar".

2) Delphi relative analysis is a method whereby consensus can be reached through collective communication in an anonymous manner. In the process, the experts are able to keep independent opinions, free from the impact of external factors.

\section{B. Quantitative Assessment Process}

Step 1: The relative importance degree of $N$ dimension $(1,2, \cdots, j)$ comprehensive evaluation index of regional resources and environmental carrying capacity in different development zones is evaluated quantitatively. Based on the three kinds of States, "familiar", "normal" and "un familiar", the importance degree carries out its own weight assignment. Then, the relative importance of the two factors in the formation of matrix $A$, where $A_{i j}$ is the relative importance of factor i relative to factor $j$, can be reduced by the order of 7,5 , $3, \ldots 1 / 5,1 / 7$ et al.;

Step 2: According to the characteristic equation $A X$ $=\lambda_{\mathrm{MAX}} X$, the feature vector $X$ is obtained. In the physical sense, all information of the matrix $A$ responsed is projected onto the plan reflected by feature vector $X$ in the largest change proportion of $\lambda_{\mathrm{MAX}}$, and all the logical relations between the $N$ dimensional influence factors are presented in the form of column vectors;

Step 3: Normalized processing of the feature vector $X$, the $\mathrm{N}$ dimensional influence factors in the same scale is quantitatively described;

Step 4: Then the quantitative assessment feedback is given to anonymous experts. A second cycle assessment based on the comprehensive consideration of the evaluation results is made, and the above three steps are repeated. Under normal circumstances, after more than three cycles, mature expert opinion can be got.

\section{PRELIMINARY ASSESSMENT}

\section{A. Results of Quantitative Assessment}

On the basis of Delphi relative analysis, through the polycyclic specialist evaluations, the relative importance of various resources and environmental capacity indexes in optimized, crucial, restricted and forbidden development zones proposed in the Planning are quantified and evaluated respectively in the paper. The final result is shown in the following Table I.

The result shows that the optimized development zones are located in Beijing-Tianjing-Hebei, Yangtze River Delta and Pearl River Delta which work as the most important dynamo for our country's economic development. However, over the years, the energy, water resources and atmospheric environment have been affected seriously. Therefore, in the energy planning, we should pay more attention to the water shortage degree, energy consumption per unit of GDP, elasticity of energy consumption, air pollutants and so on. The quantitative weight of these four indexes respectively reaches $8.04 \%, 7.43 \%, 7.95 \%$ and $8.74 \%$.

The crucial development zones are always those with favorable physiographic conditions, abundant energy resources and great development potential. In future's energy planning, we should focus more on the exploitation intensity, potential and layout of local land. Meanwhile, the key problems of regional energy development also include how to make good use of abundant water and energy resources to provide sustainable energy for the social and economic development and how to deal with the industrial waste water after the large-scale development of factories and mines. The quantitative weight value of land exploitation intensity, available water resources, water shortage degree and energy resources respectively reaches $7.25 \%, 7.42 \%, 8.03 \%, 8.73 \%$.

Both the restricted development zones and forbidden development zones are those with poor resources and environmental capacity but significant ecological security and value. They focus on the exploitation and protection of farmland and forestry, available water resources, water environment degree and the fragile ecology. All of these quantitative weights are more than $8 \%$.

\section{B. The Key Problems in the $13^{\text {th }}$ Five-Years and the Medium and Long Term Energy Development Structure}

\section{1) Sustainable supply capacity of energy resources}

The characteristics of our energy resources can be summarized as 'rich in coal, poor in oil and lack of gas' and the per captia energy resources are also insufficient. For a long time, the requirement for the energy supply is to passively meet the rigid demand while not to control the energy consumption. Both the extensive exploitation mode and the low-level developing transformation technology have led to the serious waste of our energy resources. At present, 
the effective utilization of energy resources has become one of the most concerned problems about energy planning. The“one link ,two control" policy (link the energy consumption to GDP, control the total amount and intensity of energy consumption) will be gradually practiced in energy exploitation, transformation, transportation and consumption. The transformation from passively meeting the rigid demand into moderately meeting reasonable demand should be a new manner to develop energy and also a key to maintain the sustainable supply capacity of our energy resources.

TABLE I: QUANTITATIVE ASSESSMENT

\begin{tabular}{|c|c|c|c|c|c|}
\hline System level & Index level & $\begin{array}{c}\text { Optimized } \\
\text { development } \\
\text { zone }\end{array}$ & $\begin{array}{c}\text { Crucial } \\
\text { development zone }\end{array}$ & $\begin{array}{c}\text { Restricted } \\
\text { development zone }\end{array}$ & $\begin{array}{l}\text { Forbidden development } \\
\text { zone }\end{array}$ \\
\hline \multirow{8}{*}{$\begin{array}{l}\text { Resources } \\
\text { system }\end{array}$} & Farmland exploitation intensity & $4.63 \%$ & $6.90 \%$ & $9.31 \%$ & $2.96 \%$ \\
\hline & Forestry exploitation intensity & $6.03 \%$ & $6.55 \%$ & $9.31 \%$ & $9.38 \%$ \\
\hline & Land exploitation intensity & $6.56 \%$ & $7.25 \%$ & $7.45 \%$ & $9.87 \%$ \\
\hline & Available water resources & $6.12 \%$ & $7.42 \%$ & $7.73 \%$ & $9.67 \%$ \\
\hline & Water shortage degree & $8.04 \%$ & $8.03 \%$ & $5.59 \%$ & $8.39 \%$ \\
\hline & $\begin{array}{c}\text { Energy consumption per unit of } \\
\text { GDP }\end{array}$ & $7.43 \%$ & $6.29 \%$ & $6.98 \%$ & $3.46 \%$ \\
\hline & Energy resources storage & $4.37 \%$ & $8.73 \%$ & $4.66 \%$ & $4.15 \%$ \\
\hline & Elasticity of energy consumption & $7.95 \%$ & $6.11 \%$ & $4.93 \%$ & $3.26 \%$ \\
\hline \multirow{7}{*}{$\begin{array}{l}\text { Environment } \\
\text { al system }\end{array}$} & Total emission of PM2.5 & $8.74 \%$ & $6.90 \%$ & $5.68 \%$ & $4.84 \%$ \\
\hline & Total emission of $\mathrm{CO} 2$ & $8.74 \%$ & $7.42 \%$ & $6.05 \%$ & $5.92 \%$ \\
\hline & Total emission of $\mathrm{SO} 2$ and $\mathrm{NO}_{\mathrm{X}}$ & $8.74 \%$ & $6.55 \%$ & $5.40 \%$ & $5.23 \%$ \\
\hline & $\begin{array}{l}\text { Industrial waste water treatment } \\
\text { capacity }\end{array}$ & $6.29 \%$ & $6.99 \%$ & $5.12 \%$ & $5.92 \%$ \\
\hline & Lowest water quality grade & $6.56 \%$ & $5.24 \%$ & $6.70 \%$ & $8.79 \%$ \\
\hline & Fragile ecology & $4.81 \%$ & $4.37 \%$ & $7.73 \%$ & $8.98 \%$ \\
\hline & Frequency of natural disasters & $4.98 \%$ & $5.24 \%$ & $7.36 \%$ & $9.18 \%$ \\
\hline
\end{tabular}

\section{2) Atmospheric environmental capacity}

China is still in the stage of rapid development of industrialization and urbanization, whose energy system features "Three Highs" (high intensity, high pollution and high emission). The massive discharge of pollutants has brought the persistent and accumulative damages for the atmospheric environment and now has reached the environmental critical point. In the other hand, China proposed that the carbon emission will reach the peak around 2030 and the proportion of non-fossil energy in primary energy will also be raised to $20 \%$, which puts forward higher requirements for China's energy developing mode. Therefore, the emission control of air pollutants and carbon emissions should be included in the energy planning system and then link the economic and social development to the atmospheric environmental capacity.

\section{3) Overall utilization of water resources}

China's energy structure is coal-based and the strongest constraint to the exploitable installed capacity of coal bases is water resources. At present, hydroelectricity is the most important non-fossil generating sources, which is of great importance to achieve the goals of non-fossil energy development and carbon emission. However, we should also take into consideration other aspects such as irrigation, shipping, immigration and so on. Obviously, overall consideration and utilization of water resources is one of the most essential problems for China's energy development. The water resources of energy bases is crucial to some problems such as the trade-off of various industries' technologies such as comprehensive balance, machine set cooling, etc.

\section{4) Upgrade for energy allocation mode}

Recently, at the United Nations Climate Summit in New York, Comrade $\mathrm{Xi}$ Jinping proposed to explore the construction of global energy Internet and drive to meet the global demand for electricity in a clean and green way. In the future, the traditional fossil energy including coal, petroleum and natural gas will gradually becomes exhaustible and the development and construction of large clean energy base will be increased day by day, which will present new requirements for the development of energy allocation system: firstly, the supply-side should be based on clean energy and the energy transportation net should meet the demands of large-scale exploitation, transportation and effective utilization of clean energy; secondly, try to achieve the auto-nomization of energy users, meet the requirement of mutual interaction between users and transportation net, the access need of large amount of small-sized and supply-side distributed generations and also the new electricity service demand such as the electric vehicles and smart power. As the main link of customer-side and supply-side, the characteristics of future's energy development means the traditional energy transmission and distribution network composed of railway, pipeline and highway is facing with the transformation and upgrade.

\section{5) Transformation from demand-side management to demand-side response}

Energy Internet becomes more and more popular and the mutual interaction between energy flow and information flow will help to transform the demand-side management to the demand-side response. The demand-side management focuses on controlling the users' energy consumption through some means other than the market price while the demand-side response focuses on adopting the market price to lead the users to make beneficial adjustments about the response time and standard under the base of energy informative platform. Both aspects can be connected or separated. The Third Plenary Session of the 18th Central Committee of the CPC claimed that the market should play a decisive role in the allocation of resources, which accelerates 
the development of demand-side response and the function of the energy and information platform will become increasingly outstanding.

\section{CONCLUSION}

In view of the basic ideas about land exploitation from the Planning, this paper initially analyzes the basic requirements and principles of energy development, then constructs the synthesis evaluation system of regional resources and environmental capacity and also covers fifteen refinement indexes which are derived from six elements including land resources, water resources, energy resources, atmospheric environment, water environment and ecological environment. In addition, Delphi relative analysis method is proposed to quantify and evaluate each indexes' impact on the energy development pattern.

Finally, the advices presented in this paper is useful in the medium and long term energy development structure mainly including sustainable supply capacity of energy resources, atmospheric environmental capacity, overall utilization of water resources, reasonable layout of energy transportation and demand-side response based upon energy flow and information flow platforms. This subject research serves as a reference for China's energy planning.

\section{REFERENCES}

[1] Chinese Government. The Main Functional Region Planning of China. (December 2012). [Online]. Available: http://www.gov.cn

[2] Chinese Government. Energy Development Planning in the 12th Five-Year Period (in Chinese). (January 24, 2013). [Online]. Available: http://www.gov.cn

[3] S. Chen, B. Mulgrew, and P. M. Grant, "A clustering technique for digital communications channel equalization using radial basis function networks," IEEE Trans. on Neural Networks, vol. 4, pp. 570-578, July 1993.
[4] Z. M. Jiang, "Reflections on energy issues in China (in Chinese)," Journal of Shanghai Jiaotong University, vol. 42, no. 3, pp. 345-359, 2008.

[5] C. Steven and M. Arun, "Opportunities and challenges for a sustainable energy future," Nature, vol. 488, no. 7411, pp. 294-303, 2012.

[6] Z. Y. Liu, "Smart grid and the third industrial revolution (in Chinese)," Science and Technology Daily, December 5, 2013.

[7] EREC. Energy Evolution: A Sustainable Global Energy Outlook [EB/OL]. Brussels, Belgium: ERE. (February 2012). [Online]. Available: http://www.greenpeace.org

[8] SGCC, Energy Development Status and Power Grid Function in the 12th Five-Year Period, Beijing, 2011.

[9] NEA, Report on Chinese Energy Strategy to the Year of 2030 (in Chinese), Beijing, 2010.

[10] X. P. Zhang, "Temporal-spatial characteristics of energy consumption in China and its determinants since 1990s," China Population, Resources and Environment, vol. 15, no. 2, pp. 38-41, 2005.

[11] D. M. Chen, R. Zhang, and Z. X. Tan, "Total factor energy efficiency and regional economic convergence in China: An empirical analysis based on dynamic panel data model," China Population, Resources and Environment, vol. 22, no. 1, pp. 132-137, 2012.

[12] J. Guo, L. Wang, and P. Sun, "The analysis of factors affecting regional energy consumption intensity in China," Resources Science, no. 2, pp. 31-39, 2009.

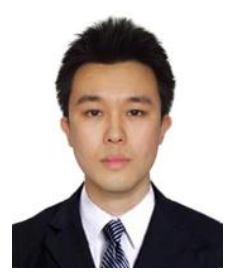

Xiaoqing Yan was born in 1986, Tianjin, China. He got his doctor degree in North China Electric Power University in 2013. His research interests are power system relay protection and control.

He is working in the State Grid Energy Research Institution of China. His research includes the power system planning, power balance analysis etc. Some of his research had been published in journal and report.

Xinting Huang was born in Anhui province, China. She received her MSc degree in finance from Warwick University in 2012. Currently, she is with China Power Finance Co., Ltd. Her main research interest is risk evaluation in energy and power department.

Shengyu Wu was born in Jiangxi province, China. She received her $\mathrm{PhD}$ degree in electrical engineering from Tsinghua University in 2010. Currently, she is with the State Grid Energy Research Institute. Her main research interest is energy and power development strategy and planning. 